\title{
APROVECHAMIENTO DE RESIDUO DE BIOMASA FÚNGICA EN LA OBTENCIÓN DE UN SISTEMA MAGNÉTICO NANOESTRUCTURADO PARA REMOVER ARSÉNICO DEL AGUA
}

\author{
Waste management of fungal biomass for the obtainment of a nanostructured magnetic system to remove arsenic from water
}

\author{
Vania Isabel MARTÍNEZ-PEÑA ${ }^{1}$, Anna ILYINA ${ }^{2 *}$, Vicente de Paul ÁLVAREZ-REYNA ${ }^{1}$, \\ Rodolfo RAMOS-GONZÁLEZ ${ }^{3}$, Juan Carlos CONTRERAS-ESQUIVEL ${ }^{2}$, \\ Mónica Lizeth CHÁVEZ-GONZÁLEZ ${ }^{2}$ y Jesús VÁSQUEZ-ARROYO ${ }^{1,4 * *}$
}

\footnotetext{
${ }^{1}$ Universidad Autónoma Agraria Antonio Narro. Periférico y Carretera a Santa Fe, Torreón, Coahuila, México. CP 27400

${ }^{2}$ Facultad de Ciencias Químicas, Universidad Autónoma de Coahuila, Blvd. V. Carranza e Ing. J. Cárdenas V., Col. República, Saltillo, Coahuila, México. CP 25280

${ }^{3}$ CONACYT-Universidad Autónoma de Coahuila, Blvd. V. Carranza e Ing. J. Cárdenas V., Col. República, Saltillo, Coahuila, México. CP 25280

${ }^{4}$ Facultad de Ciencias Químicas, Universidad Juárez del Estado de Durango, Unidad Gómez Palacio. Av. Artículo 123 s/n, Gómez Palacio, Durango, México. CP 34010

Autores para correspondencia:* annailina@uadec.edu.mx; ** jesus.vasquez@uaaan.mx
}

(Recibido: agosto 2018; aceptado: enero 2020)

Palabras clave: Aspergillus niger, quitosán, nanopartículas magnéticas, remoción de arsénico

\section{RESUMEN}

La aplicación de hongos filamentosos en procesos biotecnológicos conduce a la acumulación de biomasa como residuo. Sin embargo, la biomasa fúngica contiene diversos biopolímeros entre los cuales se encuentra la quitina -el precursor del quitosán. En el presente trabajo la biomasa fúngica se utilizó como materia prima para la extracción del biopolímero que contiene quitosán mediante el método ácido/alcalino. Se aplicaron diferentes condiciones de extracción: la concentración de álcali, el tiempo y la temperatura de los tratamientos. El biopolímero fue caracterizado y aplicado en la síntesis de ferrita de níquel por el método de coprecipitación, demostrando la posibilidad de obtener un sistema magnético nanoestructurado recubierto con quitosán fúngico en una sola etapa. Las propiedades del material magnético nanoestructurado obtenido se evaluaron por difracción de rayos-X, magnetometría de muestra vibrante y espectrometría infrarroja por transformada de Fourier. Se demostró que el material magnético nanoestructurado recubierto con quitosán fúngico remueve el $100 \%$ del arsénico presente en muestras de agua proveniente de un pozo de la Comarca Lagunera (estados de Durango y Coahuila, México), así como otros iones no nocivos para la salud. Se comprobó que la capacidad para remover el arsénico se conserva en al menos 15 ensayos. Los resultados del presente trabajo demuestran la posibilidad de aprovechar el residuo de biomasa fúngica, actualmente poco utilizado, para el diseño de nanomateriales magnéticos con impacto en la eliminación de contaminantes tóxicos del agua mediante la remoción magnética.

Key words: Aspergillus niger, chitosan, magnetic nanoparticles, arsenic removal 


\begin{abstract}
Application of filamentous fungi in biotechnological processes leads to the accumulation of biomass as waste. However, it is known that the fungal biomass contains various biopolymers among which is chitin -the precursor of chitosan. In the present work, fungal biomass was used as raw material for the extraction of a biopolymer containing chitosan by the acid/alkaline method. Different extraction conditions were assayed: the concentration of alkali, time, and temperature of treatments. The biopolymer was characterized and applied in the synthesis of nickel ferrite by the coprecipitation method demonstrating the possibility of obtaining a nanostructured magnetic system coated with fungal chitosan in a single step. The properties of the obtained nanostructured magnetic material were evaluated by X-ray diffraction, vibrating sample magnetometry, and Fourier-transform infrared spectroscopy. It was demonstrated that the nanostructured magnetic material coated with fungal chitosan removes $100 \%$ of the arsenic present in a sample from a well of Comarca Lagunera (states of Durango and Coahuila, Mexico), as well as other ions that are not harmful to health. It was found that the capacity to remove arsenic is conserved in at least 15 tests of magnetic separation of this toxic pollutant. The results of the present work demonstrate the possibility of taking advantage of the fungal biomass residue, currently little used, for the design of magnetic nanomaterials with impact on the elimination of water from toxic pollutants by magnetic separation.
\end{abstract}

\section{INTRODUCCIÓN}

Actualmente los hongos filamentosos se aplican en diferentes procesos biotecnológicos para la obtención de productos con alto valor agregado (Osuna 2013, Santos-Domínguez et al. 2017). La biomasa fúngica generada en diferentes procesos frecuentemente se considera como un residuo que puede contaminar el ambiente al no someterse al tratamiento adecuado. En diversas investigaciones realizadas se muestra la posibilidad de utilizar la biomasa fúngica para eliminación de contaminantes en efluentes acuosos (Gómez-Bertel et al. 2008, Santos-Domínguez et al. 2017). La capacidad de adsorber los iones, As (V) por ejemplo, se relaciona con la composición de la pared celular que contiene biopolímeros (Santos-Domínguez et al. 2017) que pueden interactuar con los compuestos iónicos.

Uno de los biopolímeros que se encuentran en la pared celular de hongos es la quitina. A partir de la quitina fúngica se puede obtener quitosán. Comúnmente se utiliza el método ácido/alcalino: la fracción insoluble en el medio básico se precipita, se separa por centrifugación y se trata con un medio ácido para solubilizar el quitosán. A partir de la solución obtenida mediante la precipitación con un álcali, se obtiene una fracción enriquecida con quitosán (Pochanavanich y Suntornsuk 2002, Kumaresapillai et al. 2011, Muñoz et al. 2015). Los procedimientos de extracción (Pochanavanich y Suntornsuk 2002, Kumaresapillai et al. 2011, Muñoz et al. 2015) difieren en las concentraciones de hidróxido y ácido, así como en temperatura y periodo de tratamiento. Por lo que, con cada nueva fuente microbiana, es apropiado probar diferentes condiciones para seleccionar las que conducen a un mayor porcentaje de extracción.

El uso de residuos agroindustriales y biotecnológicos como parte de tratamiento de contaminación ambiental presentan un gran interés debido a que los biopolímeros provienen de fuentes renovables y pueden ser modificados química y enzimáticamente para obtener los grupos funcionales apropiados para la interacción con los compuestos que se pretende extraer (Pokhrel y Viraraghavan 2006, Iliná et al. 2009). El arsénico es un elemento tóxico y su eliminación se presenta como un reto para los investigadores.

El arsénico es un metaloide no degradable, que se encuentra en la naturaleza en diferentes estados de oxidación, los más frecuentes son - III (arsinas), 0 (arsénico), + III (arsenito), + V (arseniato) (Oremland y Stolz 2003). El nivel elevado de arsénico encontrado en agua, principalmente como arsenito y arseniato, es la principal causa de intoxicación por arsénico en el mundo (Guha-Mazumde 2008).

Alrededor de 30 países han reportado problemas de contaminación de agua por arsénico, entre los cuales se encuentran Taiwán, Chile, India, México y Bangladesh (Moran-Martínez y García-Salcedo 2016). En México se ha detectado la presencia de arsénico en cuerpos subterráneos de agua en estados como Coahuila, Durango, Guanajuato, Zacatecas y Chihuahua, donde se alcanzan concentraciones de arsénico hasta de $1.0 \mathrm{mg} / \mathrm{L}$ (IMTA 1998). Es importante destacar que la ingesta de arsénico inorgánico incluso en concentraciones bajas $(0.010 \mathrm{ppm})$, puede 
causar a largo plazo enfermedades como cáncer de vejiga, hígado y riñón. La ingesta de arsénico se ha relacionado con otros efectos sobre la salud, por ejemplo en el sistema reproductivo y en el desarrollo embrionario, con enfermedades cardiovasculares, pulmonares y lesiones de piel (George et al. 2006).

Se han utilizado diversas tecnologías para eliminar el arsénico del agua subterránea. Las más comúnmente utilizadas incluyen coprecipitación con alumbre o hierro, adsorción sobre flóculos coagulados, resinas de intercambio iónico, ósmosis inversa y técnicas de membrana (Parga et al. 2005). Estas y otras tecnologías tienen sus deficiencias, por ejemplo, la vida útil de algunas membranas y resinas es muy corta, en algunos casos se necesita el reemplazo de estas después de cuatro o cinco usos, aunado a que estas técnicas son muy costosas y requieren operación y mantenimiento de alta tecnología (Parga et al. 2005). Otra desventaja que presenta la mayoría de estas tecnologías es la alta producción de residuos tóxicos, los cuales por su origen no pueden biodegradarse y presentan un problema para su confinamiento (USEPA 2000).

El desarrollo de nanotecnología conduce a la obtención, caracterización y aplicación de nanomateriales con elevada capacidad de adsorción de iones tóxicos (Zhang et al. 2013). Los nanosistemas magnéticos permiten diseñar una novedosa técnica de extracción de contaminantes mediada por la separación magnética, en la cual el adsorbente puede ser adicionado a la corriente del efluente y ser recuperado posteriormente con un campo magnético externo. Los nanomateriales de óxidos de hierro adsorben los compuestos de arsénico del agua con relevante eficiencia. Las ferritas son un grupo de materiales óxido magnéticos de gran importancia tecnológica. Dentro de este grupo se encuentran las ferritas de níquel $\left(\mathrm{NiFe}_{2} \mathrm{O}_{4}\right)$. Una ventaja de los nanomateriales de $\mathrm{Fe}$ es que no son tan costosos y son fáciles de producir y manipular.

Para estabilizar las nanopartículas se ha propuesto el uso de biopolímeros, los cuales ayudan a mantener las estructuras nanométricas y además facilitan la captación de iones metálicos en el agua debido a la presencia de diversos grupos funcionales (Osuna et al. 2001). Los biopolímeros de microalgas, alginato, quitina y quitosán son compuestos que se usan para este propósito y se destacan por su alta capacidad de remover los iones de metales pesados (Osuna et al. 2012).

Osuna et al. (2012) demostraron la posibilidad de obtener nanopartículas de magnetita recubiertas con quitosán mediante la coprecipitación en una sola etapa, a diferencia de los métodos de síntesis y funcionalización en dos etapas, mostrando la capacidad para interactuar con los cationes de $\mathrm{Pb}$ (II). A diferencia de otros biopolímeros, el quitosán es un polímero hidrófilo con grupos amino e hidroxilo, abundante en la naturaleza y no tóxico (Babel y Kurniawan 2003, Kyzas et al. 2013). Este biopolímero tiene la función de estabilizar los sistemas nanoestructurados, así como ligar y/o quelar los iones, dando lugar a la bioadsorción (Barkatta et al. 2009).

Los objetivos del presente trabajo fueron: 1) seleccionar el procedimiento para la extracción de una fracción que contiene quitosán a partir de biomasa de Aspergillus niger proveniente de un proceso de producción biotecnológica de lipasa; 2) caracterizar parcialmente el biopolímero obtenido; 3) demostrar la posibilidad de obtener en una sola etapa las nanopartículas magnéticas de ferrita de níquel recubiertas con este quitosán por medio de la técnica de coprecipitación; 4) evaluar las características de las muestras de agua obtenidas de un pozo de la Comarca Lagunera que contiene iones de arsénico, antes y después del tratamiento con los sistemas nanoestructurados, para demostrar su potencial en remoción de iones de este metaloide; 5) valorar la remoción de arsénico de las muestras de agua en varios ciclos de aplicación de los sistemas nanoestructurados.

\section{MATERIALES Y MÉTODOS}

\section{Manejo del cultivo fúngico}

Para la experimentación se empleó una cepa de Aspergillus niger, productora de la enzima lipasa, de la colección fúngica del Cuerpo Académico de Nanobiociencia de la Facultad de Ciencias Químicas de la Universidad Autónoma de Coahuila, Unidad Saltillo. La cepa fue conservada en agar papa-dextrosa (PDA) en tubos inclinados.

La obtención del cultivo se llevó a cabo de acuerdo con la metodología descrita por Smith (1991). La cepa de Aspergillus niger se inoculó y proliferó hasta la esporulación en cajas Petri con PDA por triplicado, para obtener cultivos monospóricos aislados de acuerdo con Reyes et al. (2013). Al final del séptimo día, utilizando un sacabocado de $5 \mathrm{~mm}$ de diámetro, se obtuvieron discos con esporas que se utilizaron como inóculo para la proliferación en una fermentación sumergida.

\section{Proliferación en fermentación sumergida}

La fermentación sumergida se llevó a cabo en matraces Erlenmeyer de $2 \mathrm{~L}$ que contenían $500 \mathrm{~mL}$ de medio Czapek-Dox: $0.5 \mathrm{~g} / \mathrm{L} \mathrm{KCl}, 3 \mathrm{~g} / \mathrm{L} \mathrm{NaNO}_{3}$, $1 \mathrm{~g} / \mathrm{L} \mathrm{K}_{2} \mathrm{HPO}_{4}, 0.5 \mathrm{~g} / \mathrm{L} \mathrm{MgSO}_{4} \cdot 7 \mathrm{H}_{2} \mathrm{O}, 5 \mathrm{~g} / \mathrm{L}$ glucosa. 
Se utilizaron 20 discos esporulados como inóculo inicial. Después de $24 \mathrm{~h}$ de proliferación, al medio de fermentación se le adicionó aceite de olivo (hasta $2 \% \mathrm{v} / \mathrm{v}$ ) como inductor de la síntesis de lipasas (Osuna 2013). La fermentación se llevó a cabo durante $120 \mathrm{~h}$ a $25^{\circ} \mathrm{C}$. La biomasa fue separada del medio de cultivo por filtración.

\section{Obtención de la fracción con quitosán fúngico de Aspergillus niger}

Para la selección del procedimiento apropiado para la extracción de la fracción con quitosán fúngico a partir de Aspergillus niger, se siguieron las metodologías descritas previamente (Pochanavanich y Suntornsuk 2002, Kumaresapillai et al. 2011, Muñoz et al. 2015).

Al finalizar la fermentación, el micelio fúngico se recuperó por filtración en papel filtro Whatman núm. 1. El filtrado se lavó con agua destilada y se sometió a secado a $65^{\circ} \mathrm{C}$ hasta peso constante.

La biomasa seca $(13 \mathrm{~g})$ fue tratada con $0.03 \mathrm{~L}$ de solución alcalina de $\mathrm{NaOH}$ (en ensayo 1 a $1 \mathrm{M}$, en ensayo 2 a $5 \mathrm{M}$, y en ensayo 3 a $2 \mathrm{M}$ ) en autoclave a $121{ }^{\circ} \mathrm{C}$ por $15 \mathrm{~min}$. Después del tratamiento la fracción insoluble se precipitó mediante centrifugación a $12000 \mathrm{rpm}$ por $15 \mathrm{~min}$. El precipitado se lavó con $30 \mathrm{~mL}$ de agua destilada y se centrifugó bajo las mismas condiciones.

El tratamiento ácido se realizó añadiendo al material insoluble $40 \mathrm{~mL}$ de ácido acético al $2 \%$. Siguiendo los procedimientos descritos previamente (Pochanavanich y Suntornsuk 2002, Kumaresapillai et al. 2011, Muñoz et al. 2015), el tratamiento con el ácido acético se llevó a cabo de la siguiente manera: ensayo $1,18 \mathrm{~h}$ a $40^{\circ} \mathrm{C}$, ensayo $2,12 \mathrm{~h} \mathrm{a} 40^{\circ} \mathrm{C}$, y ensayo $3,3 \mathrm{~h}$ a $95^{\circ} \mathrm{C}$ bajo agitación mecánica. La solución obtenida se centrifugó a $12000 \mathrm{rpm}$ por $15 \mathrm{~min}$. El sobrenadante se filtró por fibra de vidrio. El pH de la solución del sobrenadante se ajustó a 10 utilizando $\mathrm{NaOH} 2 \mathrm{M}$. El quitosán se precipitó mediante centrifugación a $12000 \mathrm{rpm}$ por $15 \mathrm{~min}$. El quitosán obtenido de la precipitación se lavó por 30 min con agua destilada, etanol $95 \%$ y acetona adicionando $20 \mathrm{~mL}$ de cada solvente y centrifugando bajo mismas condiciones, posteriormente se secó a $60^{\circ} \mathrm{C}$ hasta peso constante, obteniendo un polvo de color blanco grisáceo.

\section{Caracterización del quitosán fúngico} Análisis del hidrolizado mediante cromatografía de intercambio aniónico (HPAEC-PAD)

La muestra con quitosán fúngico, obtenida mediante el ensayo 1, fue sometida a hidrólisis ácida: a
$3 \mathrm{mg}$ de muestra se adicionaron $1 \mathrm{~mL} 2 \mathrm{M}$ de ácido trifluoroacético (TFA), posteriormente la mezcla se sometió a calor húmedo en autoclave por $4 \mathrm{~h}$ a $120{ }^{\circ} \mathrm{C}$ (Saeman 1945).

La muestra hidrolizada se analizó por cromatografía de intercambio aniónico de alto rendimiento con detección amperométrica pulsada (HPAEC-PAD) para la identificación de hidratos de carbono.

Se utilizó el equipo de HPAEC-PAD (Dionex ICS-5000, Modelo ED40, EUA), con columna analítica de resina anión intercambiador Carbopac PA1 $(250 \mathrm{x} 4 \mathrm{~mm})$ y una columna de protección PA Carbopac $(25 \times 3 \mathrm{~mm})$. La muestra hidrolizada se filtró con membrana de acetato de celulosa con tamaño de poro de $0.20 \mu \mathrm{m}$. El filtrado se inyectó manualmente. La cromatografía se llevó a cabo con un caudal de $0.1 \mu \mathrm{l} / \mathrm{min}$ a temperatura ambiente con un sistema de gradiente de hidróxido de sodio 5-200 mM. Los extractos se eluyeron isocráticamente con hidróxido de sodio $5 \mathrm{mM}$ durante $15 \mathrm{~min}$, incrementando la concentración hasta $200 \mathrm{mM}$ en $5 \mathrm{~min}$ y posteriormente manteniendo este nivel de concentración por $5 \mathrm{~min}$ más. Se inyectaron $100 \mu \mathrm{L}$ de estándares de azúcares y aminoazúcares (glucosa, D-glucosamina y N-acetil glucosamina) a una concentración de 20-100 ppm, el tiempo de análisis fue de $25 \mathrm{~min}$.

\section{Espectrometría infrarroja por transformada de Fourier (FTIR)}

Se analizaron tres muestras de una fracción de la biomasa fúngica de Aspergillus niger obtenidas bajo tres procedimientos para extracción de quitosán, así como la muestra de quitosán de Sigma-Aldrich (testigo) en un espectrofotómetro (Magna IR 550 de Nicolet, EUA) de acuerdo con el procedimiento estándar.

\section{Síntesis de nanopartículas magnéticas de ferrita de níquel recubiertas con quitosán}

La síntesis de las nanopartículas magnéticas de ferrita de níquel $\left(\mathrm{NiFe}_{2} \mathrm{O}_{4}\right)$ se realizó mediante el método de coprecipitación de acuerdo con Maaz et al. (2009). En la reacción se utilizó una relación 2:1 de cationes $\mathrm{Fe}^{3+}$ y $\mathrm{Ni}^{2+}$, respectivamente. Se prepararon $50 \mathrm{~mL}$ de una disolución de $\mathrm{FeCl}_{3} \times \mathrm{H}_{2} \mathrm{O}$ $0.2 \mathrm{M}$ y de $\mathrm{NiCl}_{2} \times \mathrm{H}_{2} \mathrm{O} 0.10 \mathrm{M}$ en agua bidestilada. Para la obtención de ferrita de níquel recubierta con quitosán se aplicó el procedimiento descrito por Osuna et al. (2012). A la solución de sales se adicionaron $0.040 \mathrm{~g}$ de quitosán fúngico extraído de Aspergillus niger.

Una vez disueltos los componentes, la disolución se llevó a una temperatura de $50^{\circ} \mathrm{C}$ en agitación 
constante. Posteriormente se agregaron por goteo $10 \mathrm{~mL}$ de una solución de $\mathrm{NaOH} 8 \mathrm{M}$, dejando reaccionar el sistema durante $30 \mathrm{~min}$ a la misma temperatura y con agitación constante. A la solución obtenida se le realizó cuatro lavados con agua destilada mediante centrifugación (Beckman J2-MI, EUA) a $12000 \mathrm{rpm}$ por $10 \mathrm{~min}$. Finalmente, las nanopartículas se sometieron a un tratamiento hidrotérmico en autoclave por $8 \mathrm{~h}$ a $121{ }^{\circ} \mathrm{C}$. El material obtenido se separó con un magneto y se sometió al proceso de secado a $100{ }^{\circ} \mathrm{C}$ por $24 \mathrm{~h}$ en horno de secado (Felisa ${ }^{\circledR}$ Modelo FE-291 D, México). Además, se realizó la síntesis de las nanopartículas de $\mathrm{NiFe}_{2} \mathrm{O}_{4}$ recubiertas con quitosán comercial (Sigma-Aldrich), siguiendo el procedimiento anteriormente descrito.

Caracterización de nanopartículas magnéticas de ferrita de níquel con y sin quitosán Difracción de rayos- $X(X R D)$

La difracción de rayos-X se realizó en un difractómetro (Rigaku, Ultima IV, EUA), el intervalo de barrido fue de 10 a $80^{\circ}$ en la escala 2q, la velocidad de barrido fue de $0.02 \mathrm{~s}$. La radiación utilizada fue la del cobre $\mathrm{K} \alpha$ con un valor de longitud de onda de $1.54056 \AA$ A. Se utilizaron valores de $25 \mathrm{~mA}$ y $35 \mathrm{kV}$ para la intensidad de corriente y el voltaje, respectivamente. La técnica permitió determinar las fases cristalinas presentes en los diferentes materiales obtenidos y calcular el diámetro de partícula por medio de la ecuación de Scherrer.

\section{Propiedades magnéticas}

Para determinar las propiedades magnéticas de las nanopartículas sintetizadas se utilizó un magnetómetro de muestra vibrante (VSM) marca Lake Shore modelo 7300 (EUA). Las muestras se analizaron a temperatura ambiente y el campo máximo aplicado fue de 20000 Oe. Las muestras de nanopartículas (con y sin quitosán) se analizaron en polvo y sin tratamiento previo.

\section{Determinación de grupos amino en nanopartículas magnéticas de ferrita de níquel con quitosán \\ La determinación de los grupos amino presentes en las nanopartículas magnéticas de ferrita de níquel recubiertas con quitosán se realizó con ninhidrina, de acuerdo con la técnica descrita previamente por Osuna (2013). Muestras de $0.1 \mathrm{~g}$ de nanopartículas magnéticas se dispersaron en $1 \mathrm{~mL}$ de agua desio- nizada. Posteriormente se añadieron $0.6 \mathrm{~mL}$ de una solución de ninhidrina al $0.2 \%$ disuelta en etanol al $70 \%$. La mezcla se dejó hervir por 10 min y se dejó}

enfriar por $2 \mathrm{~h}$. Se determinó la absorbancia a $570 \mathrm{~nm}$ en espectrofotómetro UV-visible (Cary ${ }^{\circledR}-50$, EUA). La curva de calibración se realizó utilizando glicina en un intervalo de concentraciones de 0 a $2 \mathrm{mM}$.

Tratamiento de agua de pozo de la Comarca Lagunera con nanopartículas magnéticas de ferrita de níquel

\section{Muestra de agua de la Comarca Lagunera}

La muestra de agua que se utilizó para los ensayos fue proporcionada por el Sistema Municipal de Aguas y Saneamiento (SIMAS) de Torreón, Coahuila.

La caracterización química (niveles de iones $\mathrm{Na}^{+}$, $\mathrm{Ca}^{2+}, \mathrm{Mg}^{2+}, \mathrm{CO}_{3}{ }^{-2}, \mathrm{HCO}_{3}{ }^{-}, \mathrm{Cl}^{-}, \mathrm{SO}_{4}{ }^{-2}$, As total y $\mathrm{pH}$ ) del agua utilizada se llevó a cabo por medio de análisis volumétricos (Loveday 1974), realizando los procedimientos aprobados por la Organización Panamericana de la Salud (Rojas 2002) practicados en el Laboratorio de Suelos de la Universidad Autónoma Agraria Antonio Narro, Unidad Laguna, para la evaluación de la calidad de agua.

\section{Tratamiento de muestra de agua con nanopartículas}

El tratamiento de la muestra de agua se realizó utilizando tres sistemas nanoestructurados: 1) nanopartículas magnéticas de ferrita de níquel, 2) nanopartículas de ferrita de níquel recubiertas con quitosán comercial (Sigma-Aldrich) y 3) nanopartículas recubiertas con quitosán fúngico extraído de biomasa de Aspergillus niger. En todos los ensayos se aplicaron $0.2 \mathrm{~g}$ de sistema magnético y $50 \mathrm{~mL}$ de agua, las condiciones de incubación fueron a temperatura ambiente de $25^{\circ} \mathrm{C}$ y agitación constante en un agitador horizontal (Thermolyne, EUA) a $250 \mathrm{rpm}$ por $6 \mathrm{~h}$. El sistema magnético fue retirado del medio acuoso por medio de un campo magnético externo.

Después de la separación de las nanopartículas con el imán, la fase acuosa fue retirada y sometida a la evaluación de arsénico en el equipo Wagtech Arsenator $^{\circledR}$ (WAG- WE10500, EUA) (SafarzadehAmiri et al. 2011).

Para evaluar la posibilidad del uso repetido de las nanopartículas, en el tratamiento de agua se utilizaron nuevas muestras de agua y las mismas nanopartículas, esto se realizó bajo las condiciones descritas anteriormente.

Las muestras de agua tratada, obtenidas después de 15 aplicaciones con las mismas nanopartículas, fueron sometidas al análisis por absorción atómica, como se describe a continuación, para corroborar los resultados de concentración de arsénico determinada en el equipo de detección de arsénico. 
Medición analítica de arsénico en el equipo diseñado para la cuantificación de arsénico (Arsenator)

La medición de la concentración de arsénico en las muestras de agua, antes y después del tratamiento con nanopartículas, se efectuó en un sistema Wagtech Arsenator $^{\circledR}$ (WAG- WE10500, EUA). El procedimiento se realizó según las especificaciones del fabricante con su equipo. La mínima concentración detectada por el equipo es de $2 \mathrm{ppb}$, la máxima es de $100 \mathrm{ppb}$ (Safarzadeh-Amiri et al. 2011).

\section{Análisis por espectrometría de absorción atómica}

Algunas de las muestras también fueron analizadas por espectrometría de absorción atómica con horno de grafito y generador de hidruros (Buck 210 VGP, Buck Scientific, EUA), conforme a las especificaciones de la NOM-117-SSA1-1994 (ISSA 1994), con un límite de cuantificación de 1 ppb. El ensayo se realizó con el propósito de comprobar los resultados obtenidos en Wagtech Arsenator ${ }^{\circledR}$.

\section{Análisis de datos}

Para el análisis de datos se utilizó estadística descriptiva considerando que todos los ensayos fueron realizados por triplicado.

\section{RESULTADOS}

\section{Extracción de quitosán a partir de Aspergillus niger}

En el cuadro I se presentan los resultados de los tres ensayos efectuados para la extracción del quitosán fúngico de Aspergillus niger. Las tres extracciones se efectuaron aplicando en cada ensayo $13 \mathrm{~g}$ de micelio seco (peso inicial). Se demostró (Cuadro I) que la mayor cantidad $(0.2 \mathrm{~g})$ y el mayor rendimiento $(1.54 \%)$ fueron obtenidos en el ensayo 1 en el cual la extracción se efectuó utilizando $\mathrm{NaOH}$ a $1 \mathrm{M}$ y el tratamiento de ácido acético fue llevado a cabo a una temperatura de $40{ }^{\circ} \mathrm{C}$ por un tiempo mayor de $18 \mathrm{~h}$. Estas condiciones fueron seleccionadas para ser aplicadas en todas las demás etapas del trabajo.

\section{Análisis del hidrolizado de quitosán fúngico por HPAEC-PAD}

El perfil cromatográfico de los carbohidratos detectados por HPAEC-PAD en el hidrolizado obtenido de la fracción extraída de biomasa de $A s$ pergillus niger, la que contiene quitosán fúngico, se presenta en la figura 1. Utilizando los estándares se identificó la presencia de glucosamina, glucosa y Nacetilglucosamina. Los tres picos correspondientes a estos carbohidratos se caracterizan por los tiempos de retención en minutos de $15.7,18.9$ y 19.35 , respectivamente. A partir de las curvas de calibración la concentración de estos carbohidratos se estimó en 7.6, 151 y $25 \mathrm{ppm}$, respectivamente.

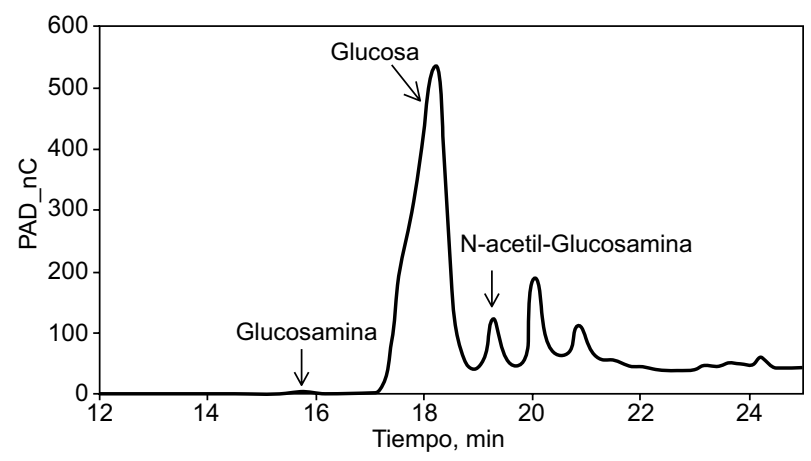

Fig. 1. Perfil cromatográfico correspondiente al hidrolizado del quitosán de Aspergillus niger

Cabe destacar que se encontró una mayor concentración de glucosa, respecto de la glucosamina y

CUADRO I. RENDIMIENTOS DE EXTRACCIÓN DE QUITOSÁN EN DIFERENTES ENSAYOS

\begin{tabular}{|c|c|c|c|c|c|c|}
\hline Ensayo & $\begin{array}{l}\text { Peso inicial } \\
(\mathrm{g})\end{array}$ & $\begin{array}{l}\text { Tratamiento con } \\
\mathrm{NaOH}(\mathrm{M})\end{array}$ & $\begin{array}{l}\text { Tiempo de tratamiento } \\
\text { con ácido }(\mathrm{h})\end{array}$ & $\begin{array}{l}\text { Temperatura de } \\
\text { tratamiento con } \\
\text { ácido }\left({ }^{\circ} \mathrm{C}\right)\end{array}$ & $\begin{array}{l}\text { Peso final (g) } \\
\text { (DE) }\end{array}$ & $\begin{array}{l}\text { Rendimiento } \% \\
\text { (DE) }\end{array}$ \\
\hline 1 & 13 & 1 & 18 & 40 & $\begin{aligned} & 0.20 \\
&( \pm 1.4 \mathrm{E}-5)\end{aligned}$ & $\begin{array}{c}1.54 \\
( \pm 0.007)\end{array}$ \\
\hline 2 & 13 & 5 & 12 & 40 & $\begin{array}{c}0.13 \\
( \pm 8.9 \mathrm{E}-4)\end{array}$ & $\begin{array}{c}1.06 \\
( \pm 0.06)\end{array}$ \\
\hline 3 & 13 & 2 & 3 & 95 & $\begin{array}{c}0.08 \\
( \pm 8 \mathrm{E}-6)\end{array}$ & $\begin{array}{c}0.63 \\
( \pm 0.01)\end{array}$ \\
\hline
\end{tabular}

$\mathrm{DE}=$ desviación estándar 
$\mathrm{N}$-acetil-glucosamina. La glucosa es común en los biopolímeros tipo glucano, mientras que la $\mathrm{N}$-acetilglucosamina se asocia con la presencia de quitina que a diferencia del quitosán contiene grupos acetilados. Esto permite considerar el extracto obtenido a partir de la biomasa de Aspergillus niger como quitosán glucano, por su alto contenido de glucosa.

\section{Espectrometría infrarroja por transformada de Fourier (FTIR)}

Los espectros obtenidos mediante la espectrometría infrarroja por transformada de Fourier, para las tres muestras extraídas de Aspergillus niger en los ensayos 1 a 3, descritos previamente, y un testigo de quitosán comercial (Sigma -Aldrich) se presentan en la figura 2. Las bandas similares observadas en los espectros del estándar comercial y las muestras extraídas de biomasa fúngica indican la presencia de quitosán en las muestras analizadas. Así, las bandas a $3414 \mathrm{~cm}^{-1}$ se asocian con la presencia de grupos hidroxilo -OH. Las bandas observadas a $2927 \mathrm{~cm}^{-1}$ corresponden a una tensión del enlace $\mathrm{C}-\mathrm{H}$. La banda a $1664 \mathrm{~cm}^{-1}$ está probablemente relacionada con la tensión del $\mathrm{C}=\mathrm{O}$, y la de $1574 \mathrm{~cm}^{-1}$ es característica para el grupo $-\mathrm{NH}_{2}$, mientras que la banda de 1423 $\mathrm{cm}^{-1}$ corresponde a la torsión del $-\mathrm{CH}_{2}$. El estiramiento simétrico $\mathrm{C}-\mathrm{O}$ se caracteriza con la banda de $1074 \mathrm{~cm}^{-1}$ y la de $1316 \mathrm{~cm}^{-1}$ se relaciona con la vibración de C-N.

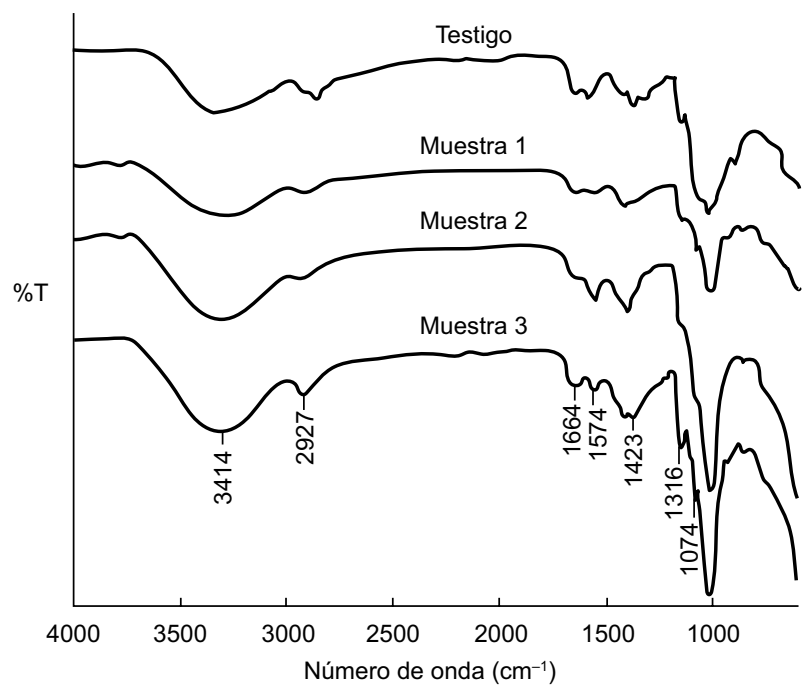

Fig. 2. Espectros de las muestras de biomasa fúngica (muestra del ensayo 1, muestra del ensayo 2, muestra del ensayo 3 (ver Cuadro I) y testigo (quitosán de Sigma-Aldrich) obtenidos por espectrometría infrarroja por transformada de Fourier
De esta manera, la caracterización de las muestras obtenidas comprueba que se logró extraer la fracción que contiene quitosán a partir de la biomasa del hongo Aspergillus niger aplicando el método álcali/ácido. La muestra obtenida mediante ensayo seleccionado con base en el mayor rendimiento se aplicó a la síntesis de nanopartículas magnéticas de ferrita de níquel para demostrar la posibilidad de obtener el sistema recubierto con quitosán glucano fúngico en una sola etapa de coprecipitación simultánea.

\section{Caracterización de nanopartículas magnéticas recubiertas con quitosán Difracción de rayos-X (XRD)}

Los tres sistemas nanoestructurados obtenidos fueron analizados por difracción de rayos-X con la finalidad de identificar su composición y tamaño de la fase cristalina, es decir, para comprobar que es ferrita de níquel y estimar el tamaño de las partículas. En la figura 3 se presentan los difractogramas correspondientes a las tres muestras $\left(\mathrm{NiFe}_{2} \mathrm{O}_{4}\right.$, de $\mathrm{NiFe}_{2} \mathrm{O}_{4}$ sintetizadas en presencia de quitosán comercial y de $\mathrm{NiFe}_{2} \mathrm{O}_{4}$ con quitosán fúngico) y el patrón de difracción (líneas verticales) de ferrita de níquel. Para las tres muestras se puede observar que todas las señales presentan en sus bases un ancho significativo y una intensidad aceptable en las reflexiones, indicando lo anterior una excelente cristalinidad y un tamaño pequeño de las partículas del material obtenido. Las principales reflexiones observadas en el patrón de

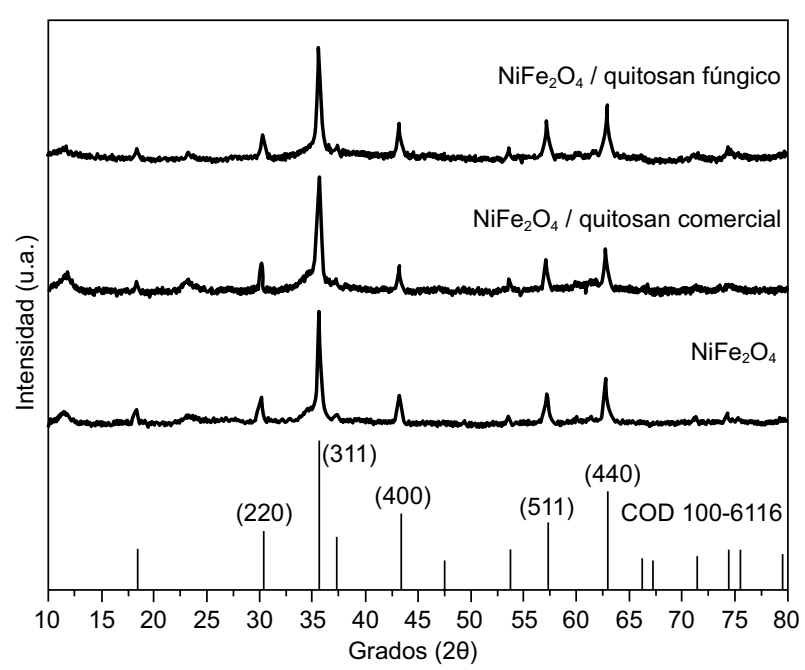

Fig. 3. Patrones de difracción de rayos-X de las muestras de $\mathrm{NiFe}_{2} \mathrm{O}_{4}$ sintetizadas y el patrón de difracción correspondiente a la ferrita de níquel, obtenido de la base de datos abierta de cristalografía (COD 100-6116 representado con líneas verticales) 
rayos-X son $30.2,35.5,43.2,57.2$ y 62.8 grados en la escala $2 \theta$, corresponden a los planos de difracción (220), (311), (400), (511) y (440), respectivamente, y pueden ser asociadas a la fase cúbica de ferrita de níquel, al comparar con el patrón de difracción \#1006116 obtenido para su indexación de la base de datos Crystallography Open Database (COD) (Blesa et al. 1993). Lo anterior comprueba la formación de ferrita de níquel por medio del método de coprecipitación tanto en presencia como en ausencia de quitosán. Así, la presencia del quitosán durante la síntesis de las nanopartículas no afecta la formación de la fase cristalina de ferrita de níquel.

A partir de los espectros de difracción de rayos$\mathrm{X}$ se pudo determinar el tamaño de la partícula de los materiales sintetizados, aplicando la ecuación de Scherrer:

$D=\frac{k \lambda}{\beta \cos \theta}$

donde:

$D$ es el tamaño promedio del cristal, $\beta$ es el ancho a la altura media de la reflexión de la muestra, $\theta$ es la posición de la reflexión, $\lambda$ es la longitud de onda de la radiación utilizada y $k$ es el factor de forma del cristal.

Para realizar el cálculo del diámetro promedio se considera la reflexión de mayor intensidad, la cual corresponde al plano (311). Para la muestra de $\mathrm{NiFe}_{2} \mathrm{O}_{4}$ se obtuvo un diámetro de $24.8 \mathrm{~nm}$, para la muestra de $\mathrm{NiFe}_{2} \mathrm{O}_{4} /$ quitosán comercial de $21.0 \mathrm{~nm}$ y para la de $\mathrm{NiFe}_{2} \mathrm{O}_{4}$ /quitosán fúngico se obtuvo un diámetro de $23.9 \mathrm{~nm}$.

De esta manera, se puede apreciar que en la síntesis de los nanomateriales se obtuvieron partículas de ferrita de níquel con el diámetro en el orden de nanómetros y la presencia del quitosán no alteró el tamaño de las nanopartículas obtenidas.

\section{Propiedades magnéticas}

Las propiedades magnéticas de las nanopartículas de $\mathrm{NiFe}_{2} \mathrm{O}_{4}$ (sin quitosán, con quitosán comercial y con quitosán fúngico) se analizaron por magnetometría de muestra vibrante (VSM). La figura 4 muestra las curvas de histéresis magnética de las muestras de ferrita de níquel analizadas y el cuadro II define los parámetros estimados a partir de estas curvas. En el cuadro II se demuestra que los valores de coercitividad para las tres muestras se encuentran entre 25 y $54 \mathrm{Oe}$, y la remanencia magnética está entre 1.5 y $3.1 \mathrm{emu} / \mathrm{g}$. Lo anterior es indicativo para concluir que las nanopartículas sintetizadas presentan un comportamiento superparamagnético. La saturación magnética presente en las muestras fue

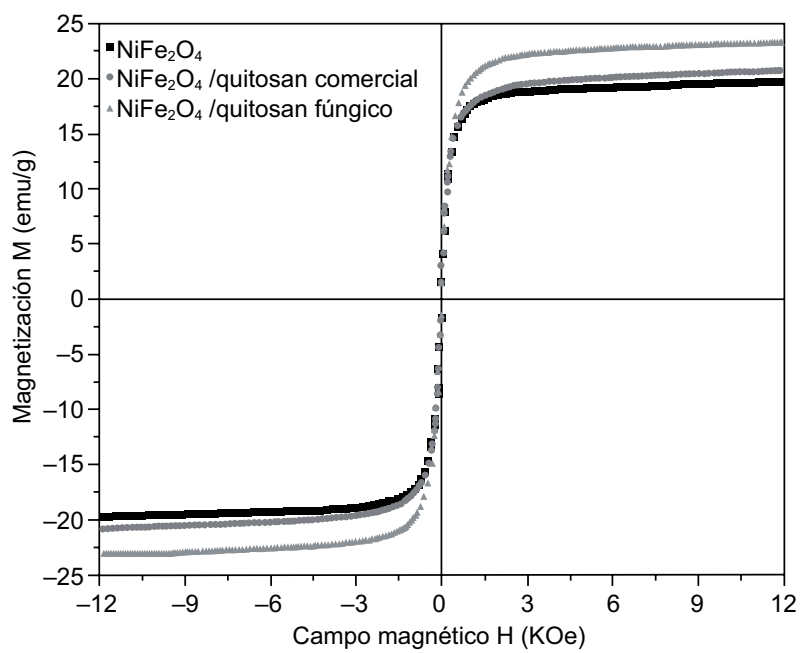

Fig. 4. Curvas de histéresis magnética de las muestras de Ni$\mathrm{Fe}_{2} \mathrm{O}_{4}$ sin o con quitosán comercial o fúngico

CUADRO II. VALORES DE COERCITIVIDAD, REMANENCIA Y SATURACIÓN MAGNÉTICADE LAS NANOPARTÍCULAS DE $\mathrm{NiFe}_{2} \mathrm{O}_{4}$

\begin{tabular}{lccc}
\hline Muestra & $\begin{array}{c}\text { Coercitividad } \\
(\mathrm{Oe})\end{array}$ & $\begin{array}{c}\text { Remanencia } \\
(\mathrm{emu} / \mathrm{g})\end{array}$ & $\begin{array}{c}\text { Saturación } \\
(\mathrm{emu} / \mathrm{g}) \mathrm{a} \\
12000 \mathrm{Oe}\end{array}$ \\
\hline $\begin{array}{l}\mathrm{NiFe}_{2} \mathrm{O}_{4} \\
\mathrm{NiFe}_{2} \mathrm{O}_{4} / \text { quitosán } \\
\text { comercial }\end{array}$ & 27.7 & 1.7 & 19.7 \\
$\begin{array}{l}\mathrm{NiFe}_{2} \mathrm{O}_{4} / \text { quitosán } \\
\text { fúngico }\end{array}$ & 53.7 & 3.1 & 20.7 \\
\hline
\end{tabular}

$\mathrm{Oe}=$ oested

en el intervalo de 19 a $23 \mathrm{emu} / \mathrm{g}$, lo cual indica que las nanopartículas pueden ser atraídas con facilidad a un campo magnético externo. De esta manera, se confirmó que las nanopartículas de ferrita de níquel muestran el comportamiento magnético.

\section{Determinación de grupos amino en nanopartículas magnéticas}

La presencia de quitosán en los sistemas nanoestructurados magnéticos se comprobó mediante la evaluación de la presencia de los grupos amino. La evaluación se realizó mediante el método espectrofotométrico que implica la reacción con ninhidrina. Considerando la concentración de los grupos amino cuantificada a partir de curva de calibración obtenida con glicina y el peso de las nanopartículas sometidas en el ensayo, se estimó que un gramo de nanopartículas magnéticas de ferrita de níquel recubiertas de 
quitosán de Aspergillus niger contiene 0.195 mmoles de grupos amino, mientras que la concentración de grupos amino en el caso del sistema con quitosán comercial fue de $0.244 \mathrm{mmol} / \mathrm{g}$.

Los resultados confirman que aplicando el método de coprecipitación simultanea de ferrita de níquel y quitosán en una sola etapa se obtuvieron las nanoparticulas superparamagnéticas de ferrita de níquel recubiertas con quitosán.

Los sistemas nanoestructurados se aplicaron para la remoción de arsénico de la muestra de agua de la Comarca Lagunera.

Tratamiento de agua de pozo de la Comarca Lagunera con nanopartículas magnéticas de ferrita de níquel

\section{Caracterización química de las muestras de agua}

Antes de realizar el ensayo entre agua contaminada con arsénico y el sistema magnético nanoestructurado de ferrita de níquel, se determinaron las características químicas de la muestra de agua de pozo de la Comarca Lagunera. Los resultados se presentan en el cuadro III. Se destaca que todos los parámetros evaluados, excepto los niveles de arsénico y cloruro, están dentro de las normas de la Organización Mundial de la Salud (OMS). El nivel de los iones de cloruro es seis veces mayor, mientras que el de arsénico es 10 veces mayor a lo permisible. Este resultado confirma que el agua de pozo sometida al estudio es una muestra que requiere un tratamiento para eliminar el arsénico.

\section{Ensayos de tratamiento de agua con nanopartículas magnéticas}

El tratamiento del agua de pozo de la Comarca Lagunera se efectuó mediante la adsorción y posterior separación magnética utilizando los tres materiales magnéticos nanoestructurados. El procedimiento fue realizado a la temperatura ambiente $\left(25^{\circ} \mathrm{C}\right)$ bajo agitación constante a $250 \mathrm{rpm}$. El tiempo de tratamiento $(6 \mathrm{~h}$ de incubación) se seleccionó con base en los resultados del estudio cinético que demostraron que a las $6 \mathrm{~h}$ de incubación el nivel de arsénico disminuyó hasta un nivel no detectado por el método aplicado reportando cero $\mathrm{mg} / \mathrm{L}$. Considerando el límite de detección de arsénico por parte del equipo utilizado, las muestras de agua fueron diluidas dos veces, por lo tanto, el nivel inicial de arsénico en todas las muestras de agua fue de $0.05 \mathrm{mg} / \mathrm{L}$.

El procedimiento de la remoción magnética de arsénico se repitió en 15 ciclos, en los cuales las nanopartículas magnéticas $(0.2 \mathrm{~g})$ se precipitaban con ayuda de un imán, el agua se decantaba y se sometía a la determinación de arsénico, mientras que al precipitado magnético se le adicionaba una nueva porción del agua $(50 \mathrm{~mL})$. En todas las muestras de agua tratada con nanopartículas magnéticas (NPM) con quitosán comercial, con quitosán fúngico y con NPM sin quitosán, al cabo de $6 \mathrm{~h}$ de incubación la concentración de arsénico disminuyó hasta niveles no detectables. Por lo tanto, se logró $100 \%$ de la remoción de arsénico en 15 ciclos de aplicación de NPM.

Las muestras de agua utilizadas en el quinceavo ciclo (con las mismas NPM aplicadas en los catorce ciclos anteriores) se sometieron al análisis de arsénico por espectrofotometría de absorción atómica con horno de grafito y generador de hidruros para corroborar el resultado de niveles de arsénico detectados con el Arsenator. El resultado obtenido por medio de este método comprobó que los niveles de

CUADRO III. CARACTERÍSTICAS DEL AGUA DE LA COMARCA LAGUNERA ANTES Y DESPUÉS DEL TRATAMIENTO CON NANOPARTÍCULAS MAGNÉTICAS DE FERRITA DE NÍQUEL (SIN Y CON QUITOSÁN) CON LA ESTIMACIÓN DEL PORCENTAJE DE REMOCIÓN DE IONES

\begin{tabular}{|c|c|c|c|c|c|}
\hline \multirow[t]{2}{*}{$\begin{array}{l}\text { Parámetro } \\
\text { químico }\end{array}$} & \multirow{2}{*}{$\begin{array}{c}\text { Niveles } \\
\text { permitidos por } \\
\text { OMS, mg/L }\end{array}$} & \multirow{2}{*}{$\begin{array}{c}\text { Antes del } \\
\text { tratamiento } \\
\mathrm{mg} / \mathrm{L}(100 \%)\end{array}$} & $\begin{array}{l}\text { NPM sin } \\
\text { quitosán }\end{array}$ & $\begin{array}{l}\text { NPM quitosán } \\
\text { comercial }\end{array}$ & $\begin{array}{l}\text { NPM quitosán } \\
\text { de } A \text {. niger }\end{array}$ \\
\hline & & & \multicolumn{3}{|c|}{ mg/L (\% de remoción) } \\
\hline $\mathrm{Na}^{+}$ & 200 & 59.8 & $3(95 \%)$ & $0(100 \%)$ & $0(100 \%)$ \\
\hline $\mathrm{Ca}^{+2}$ & 100 & 24.8 & $24(3 \%)$ & 32 (aumentó en $30 \%$ ) & $16(35 \%)$ \\
\hline $\mathrm{Mg}^{+2}$ & 70 & 1.9 & $\begin{array}{l}14.5 \text { (aumentó } \\
\text { en } 8 \text { veces) }\end{array}$ & $\begin{array}{c}8.2 \text { (aumentó } \\
\text { en } 4 \text { veces) }\end{array}$ & $\begin{array}{c}4.8 \text { (aumentó } \\
\text { en } 3 \text { veces) }\end{array}$ \\
\hline $\mathrm{CO}_{3}^{-2}$ & 500 & 2.4 & $0(100 \%)$ & $0(100 \%)$ & $0(100 \%)$ \\
\hline $\mathrm{HCO}_{3}^{-}$ & 500 & 30.5 & $0(100 \%)$ & $0(100 \%)$ & $0(100 \%)$ \\
\hline $\mathrm{Cl}^{-}$ & 5 & 30.5 & $28.4(7 \%)$ & $28.4(7 \%)$ & $21(31 \%)$ \\
\hline $\mathrm{SO}_{4}^{-2}$ & 250 & 22.2 & $12.3(45 \%)$ & $10.1(55 \%)$ & $9.4(58 \%)$ \\
\hline As (total) & 0.01 & 0.1 & $0(100 \%)$ & $0(100 \%)$ & $0(100 \%)$ \\
\hline $\mathrm{pH}$ & $6.5-8$ & 7.6 & 7.34 & 7.23 & 7.29 \\
\hline
\end{tabular}


arsénico son tan bajos que no fueron detectados por absorción atómica.

Considerando que la concentración de arsénico en el agua fue de $0.05 \mathrm{mg} / \mathrm{mL}$ y el volumen de cada muestra fue de $50 \mathrm{~mL}$, se puede calcular que en 15 tratamientos efectuados en el material nanoestructurado se adsorbieron $37.5 \mathrm{mg}$ de arsénico. Por lo tanto, considerando que se aplicaron $0.2 \mathrm{~g}$ de material, se adsorbieron $187.5 \mathrm{mg} / \mathrm{g}$ de arsénico.

Además, las muestras de agua obtenidas después de los 15 ciclos de tratamiento con materiales nanoestructurados se sometieron nuevamente a la caracterización química. En el cuadro III se presentan los resultados obtenidos. Se demostró que además de As el tratamiento conduce a la remoción completa de aniones de carbonato y bicarbonato.

El tratamiento con NPM recubiertas con quitosán comercial y fúngico conduce a una mayor disminución de la concentración de otros iones: los cationes de sodio, aniones de sulfato y iones de magnesio. La mayor remoción de cationes de calcio se observó sólo en el caso de tratamiento con NPM recubiertas con quitosán fúngico, mientras con el quitosán comercial la concentración de iones calcio se incrementó en un $30 \%$.

Una respuesta inesperada fue observada para los cationes de magnesio: la concentración se incrementó, sin embargo, el incremento es menor en presencia de quitosán, mientras que para la muestra tratada con NPM recubiertas con quitosán fúngico el aumento fue significativamente menor. Este aumento puede estar relacionado con la presencia de los iones de magnesio en las NPM como co-ion contaminante (Muzzarelli et al. 1980, Osuna et al. 2012) que se precipita bajo $\mathrm{pH}$ alcalinos (en el patrón de rayos-X obtenido (Fig. 3) se observan unos picos no identificados). De esta manera, el quitosán puede actuar como quelante de iones divalentes durante tratamientos previos y es probable que en el quinceavo ciclo se inicia su liberación, es decir que la cubierta de quitosán controla la liberación de magnesio de las NPM.

Los resultados obtenidos demuestran que las nanopartículas de ferrita de níquel sin y con quitosán pueden considerarse como un tratamiento para eliminar los iones de arsénico. El recubrimiento con quitosán conduce a que el sistema nanoestructurado magnético permita el control de otros iones en el agua. Asimismo, el quitosán fúngico muestra evidentes ventajas en la remoción de estos iones. Esta capacidad puede ser aprovechada para la disminución de la dureza del agua que presenta un problema en las aplicaciones industriales de flujos acuosos.

\section{DISCUSIÓN}

El rendimiento de extracción del quitosán obtenido en el presente trabajo (Cuadro I) es significativamente inferior a lo reportado por otros autores. Así, Muñoz et al. (2015) reportaron un rendimiento del $6.8 \%$, es decir 4.4 veces mayor al resultado del presente estudio. Kumaresapillai et al. (2011) reportaron un alto rendimiento del $26.1 \%$, es decir 17 veces mayor. Los diferentes niveles de rendimiento de la extracción de quitosán pueden estar relacionados con que distintas subespecies de Aspergillus niger contienen en su pared celular diferentes cantidades de quitina. El quitosán se obtiene a partir de la quitina, biopolímero precursor de éste. La quitina se precipita bajo las condiciones de $\mathrm{pH}$ alcalino y se solubiliza a $\mathrm{pH}$ ácido, debido a que se lleva a cabo la hidrólisis ácida de grupos acetilamino (desacetilación) formando de esta manera el biopolímero quitosán, el cual, de manera parecida a la quitina, es soluble en fase acuosa a $\mathrm{pH}$ ácido y se precipita bajo $\mathrm{pH}$ alcalino.

En la pared celular de los hongos la quitina se sintetiza a partir de la $\mathrm{N}$-acetil glucosamina por la enzima quitina sintasa, que deposita los polímeros de ésta en el espacio extracelular próximo a la membrana citoplásmica. El contenido de quitina de la pared fúngica varía según el tipo de hongo. Ésta, representa del 1 al $2 \%$ del peso seco de la pared celular de las levaduras mientras que en los hongos filamentosos puede llegar del 10 al $20 \%$ (Pontón 2008). Asimismo, en la pared de las hifas de Candida albicans es tres veces más alto que en otras levaduras, mientras que en las fases miceliales de Paracoccidioides brasiliensis y Blastomyces dermatitidis es del 25 al $30 \%$ del de la fase levaduriforme (Pontón 2008).

Por otro lado, los rendimientos de quitosán pueden variar debido a que el contenido de quitina en la pared celular puede diferir en función de las condiciones de la proliferación de hongo. En el presente estudio se tomaron las condiciones de proliferación del hongo apropiadas para la síntesis de lipasas. La biomasa del hongo se consideró como material residual del proceso de la síntesis de la lipasa. Probablemente, suplementando el medio con algunos aminoácidos y vitaminas se puede estimular la síntesis de quitina y así incrementar la cantidad de este precursor del quitosán.

La caracterización del extracto obtenido se realizó por FTIR y por cromatografía de intercambio aniónico de alto rendimiento con detección amperométrica pulsada (HPAEC-PAD). Mediante la cromatografía se demostró la presencia glucosamina, glucosa y Nacetil-glucosamina (Fig. 1). Das et al. (2006) reportan 
la extracción de quitosán a partir de Entamoeba. Sin embargo, no logran distinguir entre N-acetilglucosamina y glucosamina al aplicar la técnica cromatográfica, explicando esto por una digestión incompleta de los quito-oligosacáridos analizados.

Cao et al. (2016) aplicaron la técnica HPAECPAD para la detección de N-acetil-glucosamina en polisacáridos. El tiempo de retención reportado fue muy similar a lo observado en el presente trabajo. Sin embargo, su concentración fue tres veces mayor a lo detectado en el presente estudio.

Los resultados obtenidos mediante espectrometría infrarroja por transformada de Fourier (FTIR) efectuada para la caracterización del extracto de biomasa fúngica (Fig. 2), son muy similares a los reportados por Balanta et al. (2010) para el quitosán de Aspergillus niger habiendo una ligera diferencia en el estiramiento de los grupos $-\mathrm{OH}$ y $\mathrm{C}=\mathrm{O}$. Boonsongrit et al. (2008) y Alzate et al. (2015) también reportan resultados de espectroscopía infrarroja para muestras de quitosán que tienen gran similitud a los obtenidos en el presente trabajo de investigación.

Por lo tanto, el extracto obtenido de la biomasa de Aspergillus niger generada como residuo del proceso fermentativo bajo las condiciones de la producción de lipasa, muestra la presencia de quitosán, así como de quitina y glucano.

El polisacárido obtenido fue aplicado en el proceso de obtención de ferrita de níquel recubierta con quitosán logrando obtener el sistema nanoestructurado en una sola etapa. Sus propiedades se compararon con las de nanomateriales obtenidos sin quitosán y con quitosán comercial.

Las características que debe cumplir un material magnético nanoestructurado son: tamaño nanométrico, coercitividad y remanencia magnética, tales características se evaluaron para materiales obtenidos aplicando los métodos apropiados.

La técnica de difracción de rayos-X (XRD) permitió determinar las fases cristalinas presentes en los tres materiales obtenidos (Fig. 3), comprobando la presencia de ferrita de níquel, así como el diámetro de partícula por medio de la ecuación de Scherrer (Osuna et al. 2012) demostrando el tamaño nanométrico del material: $24.8 \mathrm{~nm}$ para la muestra de $\mathrm{NiFe}_{2} \mathrm{O}_{4}, 21.0 \mathrm{~nm}$ para la muestra de $\mathrm{NiFe}_{2} \mathrm{O}_{4}$ /quitosán comercial y $23.9 \mathrm{~nm}$ para la de $\mathrm{NiFe}_{2} \mathrm{O}_{4} /$ quitosán fúngico.

Carpenter et al. (2015) obtuvieron partículas de $\mathrm{NiFe}_{2} \mathrm{O}_{4}$ con un tamaño promedio de $26.38 \mathrm{~nm}$. Maaz et al. (2009) reportaron resultados de XRD para nanopartículas de ferrita de níquel demostrando un tamaño de partícula de $28 \mathrm{~nm}$. Sivakumar et al. (2011) reportaron un tamaño de partículas de $\mathrm{NiFe}_{2} \mathrm{O}_{4}$ de $30 \mathrm{~nm}$. Por lo tanto, los nanomateriales obtenidos en el presente trabajo se caracterizan por un diámetro menor de partícula en el orden de nanómetros. Además, la presencia del quitosán conduce a una ligera disminución del tamaño de las nanopartículas obtenidas.

Una de las características atractivas de los materiales nanoestructurados obtenidas es su propiedad magnética. Las nanopartículas sintetizadas en el presente trabajo demostraron una saturación magnética (Fig. 4 y Cuadro II) que permite clasificarlos como material superparamagnético. Los resultados son muy similares a los reportados por Joshi et al. (2014), quienes describen una saturación magnética de 20.1-35.5 emu/g, tomando en cuenta que el tratamiento de sus muestras de ferrita de níquel fue por calcinación. Sin embargo, Habibi y Fakhri (2017) reportaron valores de saturación magnética más altos de 50-51 emu/g para muestras de $\mathrm{NiFe}_{2} \mathrm{O}_{4}$ con un tratamiento de calcinación a 1100 y $600{ }^{\circ} \mathrm{C}$, respectivamente. Es evidente que a estas temperaturas tan elevadas no es posible mantener la presencia del biopolímero. Sin embargo, las nanopartículas de ferrita de níquel sintetizadas en el presente trabajo con y sin quitosán mostraron un mayor valor de saturación magnética en comparación con lo reportado por Karakaş et al. (2014), quienes detectaron $1.73 \mathrm{emu} / \mathrm{g}$ para una muestra tratada a temperatura ambiente. Por lo tanto, el tratamiento en autoclave efectuado en el presente trabajo es una alternativa viable para lograr la cristalización de la ferrita de níquel. Cabe destacar que las distintas condiciones de síntesis y tratamiento como la calcinación, pueden ser razón de la variación en las propiedades magnéticas observadas.

La cantidad de grupos amino cuantificada en las NPM recubiertas con quitosán comercial y fúngico fue determinada especrofotométricamente por medio de la reacción con ninhidrina. Kuo et al. (2012) reportaron una concentración de grupos amino de 0.116 mmoles por cada gramo de partículas, mientras que Santana (2011) demostraron la presencia de 0.189 mmoles de grupos amino por gramo de partículas. El contenido de grupos amino en los sistemas magnéticos sintetizados en el presente trabajo es mayor a lo reportado en los estudios mencionados, lo que confirma la presencia de quitosán con grupos amino disponibles para interacción con iones en las muestras de agua.

De esta manera, se aprovechó el residuo de biomasa fúngica para extraer la fracción que contenía quitosán. Además, se pudo aplicar en el proceso de 
síntesis de nanopartículas magnéticas recubiertas con quitosán. Cabe mencionar, que, según nuestro conocimiento, no hay publicaciones previas que describen este tipo de procedimiento desarrollado para la obtención de $\mathrm{NiFe}_{2} \mathrm{O}_{4}$ funcionalizada con grupos activos de quitosán.

Una vez obtenido el material magnético nanoestructurado, se aplicó en ensayos para evaluar la posibilidad de su uso para la remoción magnética de arsénico. Para definir en qué condiciones se realizará el proceso, se llevó a cabo la caracterización del agua en la cual estos materiales serían empleados. Al comparar los niveles de iones presentes en el agua, con lo establecido por la OMS, la cual determina los límites permisibles de éstos para definir la calidad de agua de consumo humano, se observó que la concentración de iones de cloro, así como de arsénico, se encuentran entre 6 y 10 veces por encima de las normas establecidas (Cuadro III). La presencia de diferentes iones, así como el $\mathrm{pH}$, pueden afectar negativamente la adsorción de arsénico (Iliná et al. 2009). En este caso el pH fue de 7.6, lo que permite clasificar el agua como ligeramente alcalina.

Posterior a la caracterización del agua, se llevaron a cabo ensayos de tratamiento de muestras de agua con los tres tipos de nanopartículas magnéticas de ferrita de níquel sin y con quitosán fúngico y quitosán comercial. Con los tres adsorbentes nanoestructurados se logró una eficiencia de remoción magnética de arsénico al $100 \%$ tanto en el primer ciclo de aplicación como en los posteriores catorce ciclos de aplicación. Cabe destacar que los óxidos de fierro, en este caso ferrita de níquel, cuando entran en contacto con agua se comportan como coloides positivos y como tales tienen la propiedad de fijar por medio de adsorción los iones con carga negativa como lo son aniones de arsénico. Esta es la razón por la cual los tres diferentes sistemas ensayados mostraron una eficiencia similar en la remoción del arsénico y de los aniones de carbonato, bicarbonato y parcialmente sulfato (Cuadro III). Además, se demostró que las nanopartículas magnéticas recubiertas con quitosán (comercial o fúngico) tienen mayor porcentaje de remoción sobre cationes $\left(\mathrm{Na}^{+}\right.$ y $\mathrm{Ca}^{+2}$ ), y la capacidad del nanomaterial recubierto con biopolímero obtenido a partir del residuo de biomasa fúngica es notablemente mayor (Cuadro III). Estos resultados abren la perspectiva de uso del sistema diseñado no sólo para la remoción de arsénico sino para adsorción de otros iones, cuya presencia en altas concentraciones puede tener efectos nocivos para la salud.
Pokhrel y Viraraghavan (2006) reportaron $95 \%$ de remoción del arsénico del agua al aplicar la biomasa fúngica tratada con sales de hierro. Montero et al. (2010) describen una eficiencia de remoción de arsénico del $87 \%$ utilizando el tratamiento con quitosán. Según Muzzarelli et al. (1980), el sistema quitosán-glucano se considera uno de los mejores quelantes de iones metálicos. Además, se conoce que los compuestos de hierro son capaces de interactuar con iones de arsénico (Iliná et al. 2009).

De esta manera, el sistema de ferrita de níquel recubierto con quitosán reúne las propiedades de nanopartículas y quitosán para interactuar con los aniones y cationes obteniendo un material adsorbente con propiedades más versátiles que cada uno por separado. El uso de quitosán extraído de biomasa fúngica presenta evidente ventaja al compararsd con el quitosán comercial en términos de mayor eficiencia de remoción de contaminantes del agua de pozo de la Comarca Lagunera.

\section{CONCLUSIONES}

Los hallazgos del presente estudio indican que la biomasa de Aspergillus niger generada como residuo en el proceso biotecnológico es una fuente de biopolímero que contiene quitosán. Es posible aplicar este polisacárido para funcionalizar el nanomaterial magnético de ferrita de níquel realizando el proceso de coprecipitación simultánea en un sólo paso. Las propiedades de material son similares a las obtenidas con quitosán comercial. Las nanopartículas magnéticas de ferrita de níquel sin y con quitosán poseen características adsorbentes de arsénico en agua a $\mathrm{pH}$ ligeramente alcalino que contiene otros iones comune. Los materiales magnéticos se pueden aplicar hasta en 15 ciclos repetidos, logrando una adsorción de por lo menos $187.5 \mathrm{mg}$ de arsénico por gramo de material magnético nanoestructurado. La presencia de cloro no afecta la remoción de arsénico. Las nanopartículas magnéticas recubiertas con quitosán ayudan a disminuir la concentración de varios iones. El mayor efecto para disminuir su concentración se observó en el caso de sistema nanoestructurado funcionalizado con quitosán fúngico al comparar con quitosán comercial. Los resultados obtenidos permiten proponer una vía alternativa para el aprovechamiento de biomasa fúngica y un nuevo método de síntesis de ferrita de níquel recubierta con quitosán, la cual tiene potencial para el control de la contaminación de agua con arsénico $\mathrm{y}$ otros iones. 


\section{AGRADECIMIENTOS}

Los autores agradecen al Consejo Nacional de Ciencia y Tecnología (CONACyT) por el financiamiento otorgado para la realización del presente proyecto. Así mismo, V.I. Martínez-Peña agradece a CONACyT por el apoyo económico otorgado mediante la beca de maestría. R. Ramos-González agradece a CONACyT por el financiamiento brindado mediante el programa "Cátedras CONACyT" (Proyecto No. 729).

\section{REFERENCIAS}

Alzate L., Cuervo R. y Valencia M. (2015). Extracción y caracterización de quitosano fúngico experimental y comercial, como potencial biomaterial para aplicaciones en ingeniería de tejidos. Rev. Iberoam. Pol. 16 (2), 112-124.

Babel S. y Kurniawan T.A. (2003). Low-cost adsorbents for heavy metals uptake from contaminated water: a review. J. Hazard. Mater. 97 (1-3), 219-43.

DOI: $10.1016 / \mathrm{S} 0304-3894(02) 00263-7$

Balanta D., Grande C.D. y Zuluaga F. (2010). Extracción, identificación y caracterización de quitosano del micelio de Aspergillus niger y sus aplicaciones como material bioadsorbente en el tratamiento de aguas. Rev. Iberoam. Pol. 11 (5), 297-316.

Barkatta A., Pulvirentia A.L., Adel-Hadadia M.A., Viragha C., Senftleb F.E., Thorpeb A.N. y Grant J.R. (2009). Composition and particle size of superparamagnetic corrosion products in tap water. Water Res. 43, 33193325. DOI: 10.1016/j.watres.2009.04.048

Blesa M.C., Amador U., Morán E., Menéndez N., Tornero J.D. y Rodríguez-Carvajal J. (1993). Synthesis and characterization of nickel and magnesium ferrites obtained from $\alpha-\mathrm{NaFeO}_{2}$. Solid State Ionics. 63, 429-436. DOI: 10.1016/0167-2738(93)90140-X

Boonsongrit Y., Mueller B. y Mitrevej A. (2008). Characterization of drug-chitosan interaction by H NMR, FTIR and isothermal titration calorimetry. Eur. J. Pharm. Biopharm. 69, 388-395.

DOI: 10.1016/j.ejpb.2007.11.008

Cao L., Wu J., Li X., Zheng L., Wu M., Liu P. y Huang Q. (2016). Validated HPAEC-PAD method for the determination of fully deacetylated chitooligosaccharides. Int. J. Mol. Sci. 17 (10), 1699-1700.

DOI: $10.3390 /$ ijms 17101699

Carpenter G., Sen R., Malviya N. y Gupta N. (2015). Microwave-assisted synthesis and characterization of nickel ferrite nanoparticles. Amer. Inst. Phys. 1675 (1), 1-4. DOI: $10.1063 / 1.4929187$
Das S., Van Dellen K., Bulik D., Magnelli P., Cui J., Head J., Robbins P.W. y Samuelson J. (2006). The cyst wall of Entamoeba invadens contains chitosan (deacetylated chitin). Mol. Biochem. Parasit. 148, 86-92.

DOI: 10.1016/j.molbiopara.2006.03.002

George C.M., Smith A.H., Kalman D.A. y Steinmaus C.M. (2006). Reverse osmosis filter use and high arsenic levels in private well water. Arch. Environ. Occup. H. 61 (4), 171-175. DOI: 10.3200/AEOH.61.4.171-175

Gómez-Bertel S., Amaya B.D., Maldonado S.C., Martínez S.M.M., Quevedo H.B., Soto G.A.B. y Pedroza R.A.M. (2008) Evaluación de tres hongos lignolíticos y de Aspergillus niger como alternativa para el tratamiento de aguas residuales del curtido de pieles. Rev. Int. Contam. Ambie. 24 (3) 93-106.

Guha-Mazumder D.N. (2008). Chronic arsenic toxicity and human health. Indian J. Med. Res. 128, 436-447.

Habibi M.H. y Fakhr F. (2017). Low temperature preparation, characterization, magnetic measurements, thermal, optical, morphological and photocatalytic properties of nano-size single phase nickel ferrite $\mathrm{NiFe}_{2} \mathrm{O}_{4}$. J. Mater. Sci-Mater. El. 28, 13455-13463. DOI: $10.1007 / \mathrm{s} 10854-017-7184-\mathrm{Z}$

Iliná A., Martínez J.L., Segura P., Villarreal J.A. y Gregorio K.M. (2009). Biosorción de arsénico en materiales derivados de maracuyá. Rev. Int. Contam. Ambie. 25 (4), 201-216.

IMTA (1998). Evaluación de diversos minerales para la remoción de arsénico de agua para consumo humano. Instituto Mexicano de Tecnología del Agua. Jiutepec, Morelos, México, 10 pp.

Joshi S., Kumar M., Chhoker S., Srivastava G., Jewariya M. y Singh V.N. (2014). Structural, magnetic, dielectric and optical properties of nickel ferrite nanoparticles synthesized by co-precipitation method. J. Mol. Struct. 1076, 55-62. DOI: 10.1016/j.molstruc.2014.07.048

Karakaş Z., Boncukcuoğlu R., Karakaş I. y Ertuğrul M. (2014). The effects of heat treatment on the synthesis of nickel ferrite $\left(\mathrm{NiFe}_{2} \mathrm{O}_{4}\right)$ Nanoparticles using the microwave assisted combustion method. J. Magn. Magn. Mater. 374, 298-306.

DOI: 10.1016/j.jmmm.2014.08.045

Kumaresapillai N., Bashab R. y Sathisha R. (2011). Production and evaluation of chitosan from Aspergillus niger MTCC strains. Iran. J. Pharm. Res. 10 (3), 553-558.

Kuo C.H., Liu Y.C., Chang C.M.J., Chen J.H., Chang C. y Shieh C.J. (2012). Optimum conditions for lipase immobilization on chitosan-coated $\mathrm{Fe}_{3} \mathrm{O}_{4}$ nanoparticles. Carbohyd. Polym. 87 (4), 2538-2545.

DOI: 10.1016/j.carbpol.2011.11.026

Kyzas G.Z., Kostoglou M., Lazaridis N.K., Lambropoulou D.A. y Bikiaris D.N. (2013). Environmental friendly technology for the removal of pharmaceutical con- 
taminants from wastewaters using modified chitosan adsorbents. Chem. Eng. J. 222, 1-42.

DOI: $10.1016 /$ j.cej.2013.02.048

Loveday J. (1974). Methods for analysis of irrigated soils. Technical Communication No. 54, CSIRO Division of Soils, Commonwealth Agricultural Bureaux, Australia, 208 pp.

Maaz K., Karim S., Mumtaz A., Hasanain S.K., Liu J. y Duan J.L. (2009). Synthesis and magnetic characterization of nickel ferrite nanoparticles prepared by co-precipitation route. J. Magn. Magn. Mater. 321, 1838-1842. DOI: 10.1016/j.jmmm.2008.11.098

Montero J.A., Paredes M.J. y Morales M.C. (2010). Utilización de quitosana para la remoción de arsénico (As) del agua. Superficies y Vacío, 23 (S) 136-139.

Moran-Martínez J. y García-Salcedo J.J. (2016). Arsénico: perspectiva epidemiológica, ambiental y de salud. Fontamara. Distrito Federal, México, 193 pp.

Muñoz G., Valencia C., Valderruten N., Ruiz D.E. y Zuluaga F. (2015). Extraction of chitosan from Aspergillus niger mycelium and synthesis of hydrogels for controlled release of betahistine. React. Funct. Polym. 91-92, 1-10.

DOI: $10.1016 /$ j.reactfunctpolym.2015.03.008

Muzzarelli R., Tanfani F. y Scarpini G. (1980). Chelating film-forming, and coagulating ability of the chitosanglucan complex from Aspergillus niger industrial wastes. Biotechnol. Bioeng. 22 (4), 885-896.

DOI: $10.1002 /$ bit.260220412

Oremland R.S. y Stolz J.F. (2003). The ecology of arsenic. Sci. J. 300, 939-944.

DOI: $10.1126 /$ science. 1081903

Osuna Y., Gregorio-Jauregui K.M., Gaona-Lozano J.G., de la Garza-Rodríguez I.M., Ilyna A., Díaz Barriga-Castro E., Saade H. y López R.G. (2012). Chitosan-coated magnetic nanoparticles with low chitosan content prepared in one-step. J. Nanomater. 2012 (ID 327562), 1-7. DOI: $10.1155 / 2012 / 327562$

Osuna Y. (2013). Inmovilización de lipasas en nanopartículas magnéticas para aplicaciones biotecnológicas. Tesis de Maestría. Facultad de Ciencias Químicas. Universidad Autónoma de Coahuila. Saltillo, Coahuila, México, 111 pp.

Parga J.R., Cocke D.L., Valenzuela J.L., Gomes J.A., Kesmez M., Irwin G., Moreno H. y Weir M. (2005). Arsenic removal via electrocoagulation from heavy metal contaminated groundwater in La Comarca Lagunera México. J. Hazard. Mater. B124. 247-254. DOI: 10.1016/j.jhazmat.2005.05.017

Pochanavanich P. y Suntornsuk W. (2002). Fungal chitosan production and its characterization. Lett. Appl. Microbiol. 35, 17-21.

DOI: $10.1046 /$ j.1472-765X.2002.01118.x
Pokhrel D. y Viraraghavan T. (2006). Arsenic removal from an aqueous solution by a modified fungal biomass. Water Res. 40 (3), 549-552.

DOI: 10.1016/j.watres.2005.11.040.

Pontón J. (2008). La pared celular de los hongos y el mecanismo de acción de la anidulafungina. Rev. Iberoam. Micol. 25, 78-82.

Reyes I., González M. y López F. (2013). Un análisis del metabolismo de Aspergillus niger creciendo sobre un sustrato sólido. Rev. Mex. Ing. Quim. 12 (1), 41-56.

Rojas R. (2002). Elementos de vigilancia y control. Guía para la vigilancia y control de la calidad del agua para consumo humano. Organización Panamericana de la Salud/Centro Panamericano de Ingeniería Sanitaria y Ciencias del Ambiente (OPS/CEPIS). OPS/CEPIS/ PUB/02.79, Lima, Perú, 66 pp.

Saeman J.F. (1945). Kinetics of wood saccharification hydrolysis of cellulose and decomposition of sugars in dilute acid at high temperature. Ind. Eng. Chem. Res. 37 (1), 43-52.

DOI: $10.1021 /$ ie50421a009

Safarzadeh-Amiri A., Fowlie P., Kazi A.I., Siraj S., Ahmed S. y Akbor A. (2011). Validation of analysis of arsenic in water samples using Wagtech Digital Arsenator. Sci. Total Environ. 409, 2662-2667.

DOI:10.1016/j.scitotenv.2011.03.016

Santana S.D.F. (2011). Magnetic nanoparticles for biocatalysis and bioseparation. Tesis de Maestría. Facultad de Ciencias y Tecnología. Universidade Nova de Lisboa. Lisboa, Portugal, 109 pp.

Santos-Domínguez E.E., Vargas-Morales J.M., CárdenasGonzález J.M. y Acosta-Rodríguez I. (2017). Remoción de arsénico (V) en solución acuosa por biomasa modificada del hongo Aspergillus niger. Información Tecnológica 28 (6), 45-52.

DOI: $10.4067 / \mathrm{S} 0718-07642017000600006$

Sivakumar P., Ramesh R., Ramanand A., Ponnusamy S. y Muthamizhchelvan, C. (2011). Synthesis and characterization of nickel ferrite magnetic nanoparticles. Mater. Res. Bull. 46, 2208-2211.

DOI: 10.1016/j.materresbull.2011.09.009.

Smith D. (1991). Maintenance of microorganisms and cultured cells. 2nd ed. Academic Press. Londres, Reino Unido, 288 pp.

ISSA (1994). Norma Oficial Mexicana NOM-117SSA1-1994, Bienes y servicios. Método de prueba para la determinación de cadmio, arsénico, plomo, estaño, cobre, fierro, zinc y mercurio en alimentos, agua potable y agua purificada por espectrometría de absorción atómica. Diario Oficial de la Federación. 29 de junio de 1995.

USEPA (2000). Technologies and costs for removal of arsenic from drinking water. The United States 
Environmental Protection Agency, Office of Ground Water and Drinking Water. Arizona, EUA, 284 pp.

Zhang L., Xia W., Teng B., Liu X. y Zhang W. (2013). Zirconium cross-linked chitosan composite: preparation, characterization and application in adsorption of $\mathrm{Cr}$ (VI). Chem. Eng. J. 229, 1-8.

DOI: $10.1016 /$ j.cej.2013.05.102 